\title{
6-Hydrazinonicotinic acid hydrazide: a useful precursor for chemo- and regioselective synthesis of new heteroaryl-linked pyridinohydrazones
}

\author{
Helio G. Bonacorso*, Gisele R. Paim, Liliane M. F. Porte, Everton P. Pittaluga, \\ Marcos A. P. Martins, and Nilo Zanatta
}

Núcleo de Química de Heterociclos (NUQUIMHE), Departamento de Química Universidade Federal de Santa Maria, 97105-900, Santa Maria, RS, Brazil

E-mail: heliogb@base.ufsm.br

\begin{abstract}
In order to demonstrate the reactivity differentiation between the two dinucleophilic centers in the 6-hydrazinonicotinic acid hydrazide hydrate (1), a series of seven new 6-[(2(arylidene)hydrazinyl]nicotinohydrazides (4) was chemoselectively accessed from the reaction of 1 with seven aryl or heteroaryl aldehydes by a simple procedure in ethanol as solvent. In a subsequent study, cyclization reactions of $\mathbf{4}$ with some 4-alkoxy-1,1,1-trifluoroalk-3-en-2-ones or triethylorthoacetate were proceeded easily also in ethanol, an eco-friendly solvent, to give regioselectively nine examples of pyrazolinyl-pyridinohydrazones and one 1,3,4-oxadiazole derivative, respectively, as stable and pure compounds in good yields.
\end{abstract}

Keywords: Hydrazones, hydrazides, pyrazoles, oxadiazoles, pyridines

\section{Introduction}

Hydrazones and their derivatives constitute a versatile class of compounds in organic chemistry and they are characterized by the presence of $-\mathrm{NH}-\mathrm{N}=\mathrm{CH}-$ or $-\mathrm{NH}-\mathrm{N}=\mathrm{CR}^{1} \mathrm{R}^{2}-$ groups in their molecules. ${ }^{1,2}$ These compounds have interesting biological properties, such as anti-inflammatory, analgesic, anticonvulsant, antituberculous, antitumor, anti-HIV and antimicrobial activity. ${ }^{3-6}$ Hydrazones are also important compounds for drug design, as possible ligands for metal complexes, organocatalysis, and also for the syntheses of heterocyclic compounds. ${ }^{7,8}$ The ease of preparation, increased hydrolytic stability relative to imines, and tendency toward crystallinity are all desirable characteristics of hydrazones. Due to these positive traits, hydrazones have been under study for a long time, but much of their basic chemistry remains unexplored. On the other hand, the presence of heterocyclic rings in many synthetic hydrazones plays a main role in their pharmacological properties. ${ }^{8}$ 
Pyrazoles have been studied for over a century as an important class of heterocyclic compounds and still attract considerable attention due to the broad range of biological activities, including analgesic, antimicrobial, antiviral, anti-inflammatory, hypoglycemic, anti-hypertensive and antitumor properties. ${ }^{9-13}$ Pyridine, which also deserves attention, is not found free in nature, but its derivatives have many chemical applications, mostly in polymers and dyes, as well as in the pharmaceutical area as analgesics, anesthetics, and psychopharmacological drugs. ${ }^{14}$

The structure of 6-hydrazinonicotinic hydrazide hydrate (1) contains two nucleophilic centers: a hydrazine and a hydrazide moiety, which must have differentiated reactivity towards the electrophiles. Thus, in one of our recent studies we carried out the cyclocondensation reactions of 4-alkoxy-4-alkyl(aryl/heteroaryl)-1,1,1-trifluoroalk-3-en-2-ones with 6hydrazinonicotinic acid hydrazide hydrate, in a equimolar ratio, and obtained regioselectively a new trifluoromethylated system as 2-(1H-pyrazolyl)-5-(1H-pyrazolylcarbonyl)pyridines in good yields (Figure 1). ${ }^{15}$ However, these reaction conditions do not lead to a chemoselective cyclization at the most reactive center (hydrazine moiety).<smiles>[R]/C(=C/C(=O)C(F)(F)F)O[Tl]</smiles>

( I )

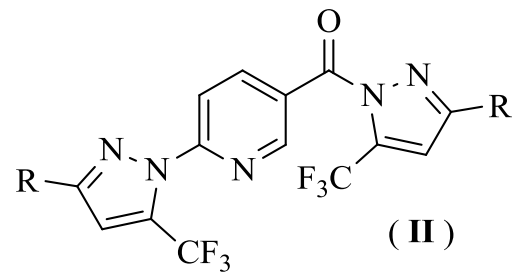

Figure 1. Generic structures of 4-alkoxy-4-alkyl(aryl/heteroaryl)-1,1,1-trifluoroalk-3-en-2-ones (I) and 2-(1H-pyrazolyl)-5-(1H-pyrazolylcarbonyl)pyridines (II).

In this context, the aim of this paper is to report the reactivity study on 6-hydrazinonicotinic acid hydrazide hydrate and to prove it as a versatile precursor for some new interesting heterocycles. Thus, attempting to demonstrate the reactivity differentiation between the two dinucleophilic centers in the hydrazide hydrate $(\mathbf{1})$, we report ( $i)$ the results of the reactions of $\mathbf{1}$ with triethylorthoacetate (Scheme 1); (ii) the synthesis of a new series of 6(alkylidenehydrazinyl)nicotinohydrazides (4) conveniently accessed from the reaction of $\mathbf{1}$ with aryl or heteroaryl aldehydes (3) (Scheme 2), and (iii) a subsequent study, where cyclization reactions of 4 with 4-alkoxy-1,1,1-trifluroalk-3-en-2-ones (5) and triethylorthoacetate are proceeded to give examples of new heteroaryl-pyridinohydrazones (6) and one 1,3,4-oxadiazole derivative (7) (Scheme 3).

\section{Results and Discussion}

\section{Synthesis and structure}

Firstly, it is well-known from our previous work ${ }^{15}$ that 4-alkoxy-1,1,1-trifluoroalk-3-en-2-ones, a 1,3-dielectrophile block, is unable to differentiate the two nucleophilic centers at the compound 
1. Thus, in an attempt to demonstrate the reactivity differentiation between the two dinucleophilic centers in the 6-hydrazinonicotinic acid hydrazide hydrate (1), we performed the reaction involving $\mathbf{1}$ and triethylorthoacetate, which was also used as the reaction solvent, at reflux $\left(145^{\circ} \mathrm{C}\right)$ for $16 \mathrm{~h}$ (Scheme 1). ${ }^{16}$ Unfortunately, no chemical differentiation was observed for compound 1 toward the cited orthoester. In this case the hydrazine and the hydrazide moieties showed similar reactivity and conducted only to the not yet known structure of 3-methyl-6-(5methyl-1,3,4-oxadiazol-2-yl)-[1,2,4]triazolo[4,3-a]pyridine (2) in $82 \%$ yield by a double [4+1] cyclocondensation reaction. The NMR data showed clearly that in this case an unexpected reactivity of the endocyclic nitrogen atom of the $\pi$-deficient pyridine ring towards the carbonyl carbon of the orthoester conducted to a geminated triazolopyridine nucleous.

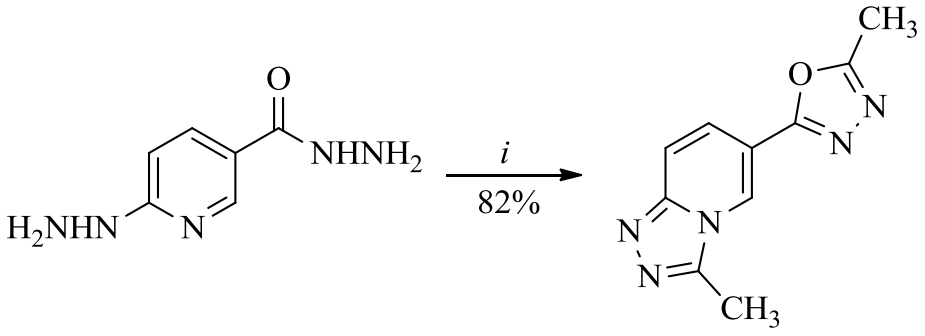

1

2

Scheme 1. Synthetic route for oxadiazolyl-triazolo-pyridine (2). Reagents and conditions: (i) $\mathrm{H}_{3} \mathrm{CC}(\mathrm{OEt})_{3}$, reflux, $16 \mathrm{~h}$.

In a second step and according to the literature, ${ }^{17-19}$ hydrazones can be obtained by refluxing ethanol or methanol as solvent, at $60{ }^{\circ} \mathrm{C}$ or at room temperature for $2-14 \mathrm{~h}$ depending on the reactivity of the carbonyl compound. Based on these literature data, we have performed several tests to obtain hydrazides (4), which varied the temperature, reaction time, solvent, and molar ratio. The most satisfactory results for the synthesis of hydrazides $(\mathbf{4 a - g})$ were obtained when we employed 6-hydrazinonicotinic acid hydrazide hydrate (1) and aryl/heteroaryl aldehydes $(\mathbf{3})$ in a molar ratio 1:1 using ethanol as solvent, at $60{ }^{\circ} \mathrm{C}$ for $7 \mathrm{~h}$ (Scheme 2). These reaction conditions allowed us for the first time to differentiate the two nucleophilic centers at $\mathbf{1}$ and to isolate the compounds 4 , in good yields $(64-94 \%)$ as stable solids by simple filtration under reduced pressure following by drying under vacuum in a desiccator containing $\mathrm{P}_{2} \mathrm{O}_{5}$. The structures of these new hydrazides (4) have then attached to the pyridine ring a stable hydrazone function and a free hydrazide function for further chemical modifications. 


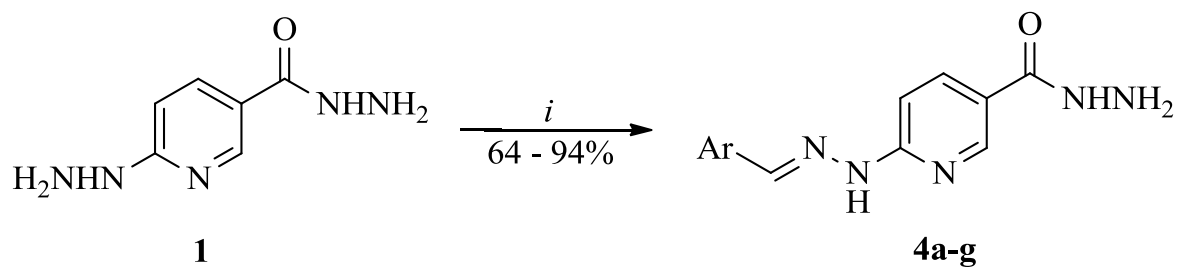

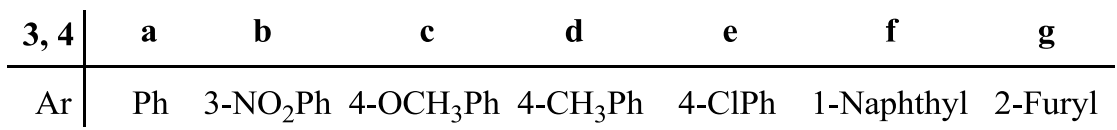

Scheme 2. Synthesis of hydrazinyl-nicotinohydrazide (4a-g). Reagents and conditions: $(i)$ ArCHO (3a-g), EtOH, $60{ }^{\circ} \mathrm{C}, 7$ h.

In an attempt to promote the synthesis of new heterocycles we have performed two types of cyclocondensation reactions, namely, [3+2] and [4+1] at the hydrazide function of compounds 4. Thus, initially, a variety of 4-alkoxy-1,1,1-trifluroalk-3-en-2-ones (5) was used to promote the synthesis of a new series of nine examples of 5-[(3-alkyl(aryl/heteroaryl)-5- trifluoromethyl-5hydroxy-4,5-dihydro-1H-pyrazol-1-yl)carbonylpyridin-2-yl]aryl/hetero-arylaldehyde hydrazones (6) by the [3+2] cyclocondensation reactions. The reactions were based on the literature data ${ }^{15}$ and carried out in ethanol as solvent at reflux for $4 \mathrm{~h}$. After the reaction time, the heterocyles 6 were isolated by simple filtration under reduced pressure in $45-83 \%$ yields (Scheme 3 ). The $4-$ methoxy-4-aryl/heteroaryl-1,1,1-trifluoroalk-3-en-2-ones (5a-c), which are readily available CCC synthetic blocks, were prepared from trifluoroacetylation reactions of enol ethers generated in situ from the respective acetophenone dimethyl acetals (for 5a-b) or 2-acetilfuran dimethyl acetal (for 5c) with trifluoroacetic anhydride, respectively, in the presence of pyridine as described in the literature. ${ }^{20-23}$

Finally, based on the first results from this work for the synthesis of compound 2, we have employed the same reaction condition to obtain a 1,3,4-oxadiazole derivative from hydrazide (4e) by a [4+1] cyclocondensation reaction with triethylorthoacetate. This reaction condition allowed to obtain 2-(5-(5-methyl-1,3,4-oxadiazol-2-yl)-pyridin-2-yl)-4-chlorobenzaldehyde hydrazone (7e) in good yield $(64 \%)$ when the reaction was carried out in triethylorthoacetate as solvent at reflux for $16 \mathrm{~h}$.

The structures of 6-[2-(aryl/heteroarylidene)hydrazinyl)] nicotinohydrazide (4a-g) were deduced from NMR experiments and compared with NMR data from other hydrazides formerly synthesized in the literature. ${ }^{15}$ Initially, compounds 4 show the ${ }^{1} \mathrm{H}$ NMR chemical shifts of the $\mathrm{NH}_{2}$ at $\delta 4.42 \mathrm{ppm}$ (broad singlet). The chemical shifts for the $\mathrm{NH}$ protons resonate at $\delta 9.61$ and $11.24 \mathrm{ppm}$. These compounds show the $\mathrm{C} \underline{\mathrm{H}}=\mathrm{N}$ proton at $\delta 8.19 \mathrm{ppm}$. Compounds $4 \mathbf{a}-\mathrm{g}$ present also the typical ${ }^{13} \mathrm{C}$ chemical shifts of their carbons in average of $\delta 164.6$ for $(\mathrm{C}=\mathrm{O}), \delta 136.8$ $(\mathrm{C}=\mathrm{N}), \delta 158.1,147.6,140.4,119.7,105.0 \mathrm{ppm}$ (pyridine ring). 

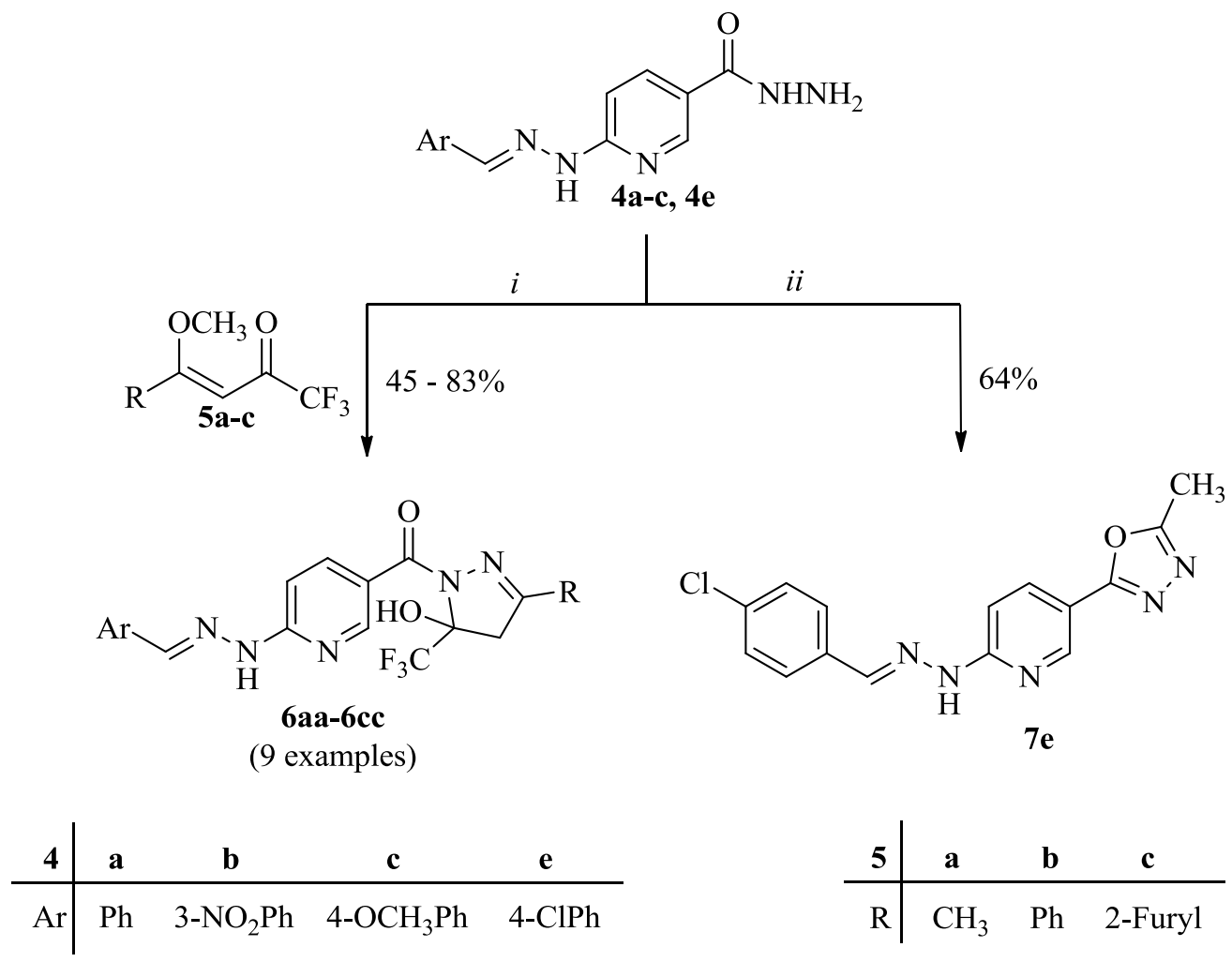

\begin{tabular}{c|ccccccccc}
$\mathbf{6}$ & $\mathbf{a a}$ & $\mathbf{a b}$ & $\mathbf{a c}$ & $\mathbf{b a}$ & $\mathbf{b b}$ & $\mathbf{b c}$ & $\mathbf{c a}$ & cb & cc \\
\hline $\mathrm{Ar}$ & $\mathrm{Ph}$ & $\mathrm{Ph}$ & $\mathrm{Ph}$ & $3-\mathrm{NO}_{2} \mathrm{Ph}$ & $3-\mathrm{NO}_{2} \mathrm{Ph}$ & $3-\mathrm{NO}_{2} \mathrm{Ph}$ & $4-\mathrm{OCH}_{3} \mathrm{Ph}$ & $4-\mathrm{OCH}_{3} \mathrm{Ph}$ & $4-\mathrm{OCH}_{3} \mathrm{Ph}$ \\
$\mathrm{R}$ & $\mathrm{CH}_{3}$ & $\mathrm{Ph}$ & $2-\mathrm{Furyl}$ & $\mathrm{CH}_{3}$ & $\mathrm{Ph}$ & $2-\mathrm{Furyl}$ & $\mathrm{CH}_{3}$ & $\mathrm{Ph}$ & 2-Furyl
\end{tabular}

Scheme 3. Synthetic route for pyrazolyl/oxazolyl-pyridine aryl/heteroaryl hydrazones $(6,7)$. Reagents and conditions: (i) EtOH, reflux, 4 h; (ii) $\mathrm{H}_{3} \mathrm{CC}(\mathrm{OEt})_{3}$, reflux, $16 \mathrm{~h}$.

Compounds 6aa-6cc show ${ }^{1} \mathrm{H}$ NMR chemical shifts of the diastereotopic methylene protons $(\mathrm{H}-4 \mathrm{a}$ and $\mathrm{H}-4 \mathrm{~b})$ as a characteristic of $\mathrm{AB}$ system and as a doublet at ca. $\delta 3.41 \mathrm{ppm}$ and another doublet at ca. $3.76 \mathrm{ppm}$, with a geminal coupling constant in the range of ${ }^{2} J=18 \mathrm{~Hz}$. The absence of signals for $\mathrm{NH}$ and $\mathrm{NH}_{2}$ protons from the hydrazide moiety of the precursors are also noted in the spectra of these compounds. The trifluoromethylated heterocycles present the typical ${ }^{13} \mathrm{C}$ NMR chemical shifts of pyrazoline ring carbons at $\delta 154.1$ (C-3), 91.7 (C-5) with a coupling constant of ${ }^{2} J=33 \mathrm{~Hz}$, at $44.8(\mathrm{C}-4)$ and at $123.4\left(\mathrm{CF}_{3}\right)$ with ${ }^{1} J=286 \mathrm{~Hz}$. The ${ }^{13} \mathrm{C}$ chemical shifts of the pyridine ring carbons present signals in the range of 104.6, 120.3, 140.8, 149.2 and $158.5 \mathrm{ppm}$. The $s p^{2}$-carbon of the hydrazone function for compounds 6 shows signals, such as singlet, in the average of $144.6 \mathrm{ppm}$. 


\section{Conclusions}

The methodologies presented in this work allowed a chemical differentiation (chemoselectivity) of the $\mathrm{N}$-reactivity in the precursor dinucleophilic functions (1). Furthermore, a facile and useful regioselective synthesis to obtain new hydrazides (4) was accomplished from the reaction of 1 with a broad scope of the selected aldehydes (3). We also demonstrated that hydrazides (4) can be used as the synthetic intermediates in [3+2] and [4+1] cyclocondensation reactions with two electrophilic molecules such as trifluoromethylated vinyl ketones $\mathbf{5}$ and triethylorthoacetate to isolate interesting new heterocyclic systems as pyrazolyl- and oxadiazolyl-pyridines (6) and (7), respectively.

\section{Experimental Section}

General. Unless otherwise indicated, all common reagents and solvents were used as obtained from commercial suppliers without further purification. All melting points were determined on a Reichert Thermovar apparatus. ${ }^{1} \mathrm{H}$ and ${ }^{13} \mathrm{C}$ NMR spectra were acquired on a Bruker DPX 200 spectrometer $\left({ }^{1} \mathrm{H}\right.$ at $200.13 \mathrm{MHz}$ and ${ }^{13} \mathrm{C}$ at $\left.50.32 \mathrm{MHz}\right)$ and Bruker DPX $400\left({ }^{1} \mathrm{H}\right.$ at 400.13 $\mathrm{MHz}$ and ${ }^{13} \mathrm{C}$ at $100.61 \mathrm{MHz}$ ) spectrometer, $5 \mathrm{~mm}$ sample tubes, $298 \mathrm{~K}$, digital resolution \pm 0.01 ppm, in DMSO- $d_{6}$, using TMS as internal reference $\left({ }^{1} \mathrm{H}\right.$ and $\left.{ }^{13} \mathrm{C}\right)$. The $\mathrm{CHN}$ elemental analyses were performed on a Perkin Elmer $2400 \mathrm{CHN}$ elemental analyzer (São Paulo UniversityUSP/Brazil).

The ESI mass spectra were performed on an Agilent 6460 Triple Quadrupole connected to a 1200 series LC and equipped with a solvent degasser, binary pump, column oven, auto-sampler, and an ESI source. The Agilent QQQ 6460 tandem mass spectrometer was operated in the positive jet stream electrospray ionization (ESI) mode. Nitrogen was used as nebulizer, turbo (heater) gas, curtain gas, and collision activated dissociation gas. The capillary voltage was set at $+3500 \mathrm{~V}$ and nozzle voltage was at $+500 \mathrm{~V}$. The ion source gas temperature was $300{ }^{\circ} \mathrm{C}$ with a flow rate of $5 \mathrm{~L} / \mathrm{min}$. The jet stream sheath gas temperature was $250{ }^{\circ} \mathrm{C}$ with a flow rate of 11 $\mathrm{L} / \mathrm{min}$. All samples were infused into the ESI source at a $5 \mu \mathrm{L} / \mathrm{min}$ flow rate. Data were acquired in positive mode MS total ion scan mode (mass scan range $m / z$ 50-650) and in positive MS/MS product ion scan mode. The mass spectra recorded were evaluated by the Qualitative Analysis software from Agilent Technologies.

General synthetic procedure for the synthesis of 3-methyl-6-(5-methyl-1,3,4-oxadiazol-2yl)-[1,2,4]triazolo[4,3-a]pyridine (2). A mixture of 6-hydrazinonicotinc hydrazide hydrate 1 (1 mmol) and triethylortoacetate $\left(1.1 \mathrm{~cm}^{3}, 6 \mathrm{mmol}\right)$ was stirred for $16 \mathrm{~h}$ at reflux. The excess of orthoacetate was removed by evaporation under reduced pressure. The product $\mathbf{2}$ was obtained as solid and dried under vacuum in a desiccator containing $\mathrm{P}_{2} \mathrm{O}_{5}$. 
3-Methyl-6-(5-methyl-1,3,4-oxadiazol-2-yl)-[1,2,4]triazolo[4,3-a]pyridine (2). White solid, yield $82 \%$, mp $145-147{ }^{\circ} \mathrm{C} ;{ }^{1} \mathrm{H}$ NMR (400.13 MHz, DMSO- $d_{6}$ ): $\delta 8.93$ (s, $\left.1 \mathrm{H}, \mathrm{Py}\right) ; 7.85-7.88$ (m, 1H, Py); 7.76 - 7.79 (m, 1H, Py); 2.77 (s, 3H, $\left.\mathrm{CH}_{3}\right) ; 2.61\left(\mathrm{~s}, 3 \mathrm{H}, \mathrm{CH}_{3}\right) \mathrm{ppm} ;{ }^{13} \mathrm{C} \mathrm{NMR}$ $\left(100.61 \mathrm{MHz}, \mathrm{DMSO}-d_{6}\right): \delta 163.9\left(\mathrm{C}_{\text {oxa }}-\mathrm{CH}_{3}\right) ; 161.4\left(\mathrm{C}_{\text {oxa }}-\mathrm{Py}\right) ; 148.2(\mathrm{Py}) ; 145.0\left(\mathrm{C}-\mathrm{CH}_{3}\right)$; 144.9 (Py); 123.7 (Py); 115.9 (Py); 110.3 (Py); $10.3\left(\mathrm{CH}_{3}\right)$; $9.5\left(\mathrm{CH}_{3}\right) \mathrm{ppm}$; MS (ESI) m/z: [(M + $\mathrm{H})^{+}$, 216.2]. Anal. Calcd for $\mathrm{C}_{10} \mathrm{H}_{9} \mathrm{~N}_{5} \mathrm{O}: \mathrm{C}, 55.81 ; \mathrm{H}, 4.22$; N, 32.54. Found: C, 55.43; H, 4.21; $\mathrm{N}, 32.82$.

General procedure for the synthesis of 6-[2-aryl/heteroarylmethylidenehydrazinyl]nicotinohydrazide (4a-g). A mixture of 6-hydrazinonicotinc hydrazide hydrate 1 (1 mmol) and aryl/heteroarylaldehydes 3a-g $(1 \mathrm{mmol})$ in $15 \mathrm{~cm}^{3}$ of ethanol was stirred for $7 \mathrm{~h}$ at $60{ }^{\circ} \mathrm{C}$.

Products 4a-g were obtained as solids, filtered under reduced pressure and dried under vacuum in a desiccator containing $\mathrm{P}_{2} \mathrm{O}_{5}$. Compounds $\mathbf{4 a - g}$ presented a high degree of purity and did not undergo recrystallization.

6-(2-Benzylidenehydrazinyl)nicotinohydrazide (4a). White solid, yield 92\%, mp 259 - 261 ${ }^{\circ} \mathrm{C} ;{ }^{1} \mathrm{H}$ NMR (400.13 MHz, DMSO- $\left.d_{6}\right): \delta 11.27$ (s, 1H, NH), $9.64(\mathrm{~s}, 1 \mathrm{H}, \mathrm{NH}), 8.59-8.60$ (m, 1H, Py), 8.08 (s, 1H, H), $7.67-7.72$ (m, 3H, Ph + Py), $7.37-7.45$ (m, 3H, Ph), $7.24-7.29$ (m, 1H, Py), 4.44 (s, 2H, NH ) ppm; ${ }^{13} \mathrm{C}$ NMR (100.61 MHz, DMSO-d $\left.d_{6}\right): \delta 164.7$ (CO), 158.3 (Py), $147.6(\mathrm{Py}), 140.5(\mathrm{Py}), 136.6(\mathrm{C}=\mathrm{N}), 134.9(\mathrm{Ph}), 128.8(\mathrm{Ph}), 128.6(\mathrm{Ph}), 126.1(\mathrm{Ph}), 119.8(\mathrm{Py})$, 105.2 (Py) ppm; MS (ESI) $m / z:\left[(\mathrm{M}+\mathrm{H})^{+}, 256.1\right]$. Anal. Calcd for $\mathrm{C}_{13} \mathrm{H}_{13} \mathrm{~N}_{5} \mathrm{O}: \mathrm{C}, 61.17$; $\mathrm{H}$, 5.13; N, 27.43. Found: C, 61.56; H, 5.16; N, 27.72.

6-[2-(3-Nitrobenzylidene)hydrazinyl]nicotinohydrazide (4b). Yellow solid, yield 94\%, mp $250-252{ }^{\circ} \mathrm{C} ;{ }^{1} \mathrm{H}$ NMR (400.13 MHz, DMSO-d $)$ ): $\delta 11.54$ (s, $\left.1 \mathrm{H}, \mathrm{NH}\right), 9.69(\mathrm{~s}, 1 \mathrm{H}, \mathrm{NH}), 8.62-$ 8.63 (m, 1H, Py), 8.47 (s,1H, H), $8.07-8.20(\mathrm{~m}, 4 \mathrm{H}, \mathrm{Py}+\mathrm{Ph}), 7.66-7.74(\mathrm{~m}, 1 \mathrm{H}, \mathrm{Ph}), 7.30-$ 7.35 (m, 1H,Py), 4.45 (s, 2H, NH${ }_{2}$ ) ppm; ${ }^{13} \mathrm{C}$ NMR (100.61 MHz, DMSO-d $)$ ): $\delta 164.6(\mathrm{CO})$, $158.0(\mathrm{Py}), 148.2(\mathrm{Py}), 147.6(\mathrm{Ph}), 138.0(\mathrm{Py}), 136.8(\mathrm{Ph}), 136.8(\mathrm{C}=\mathrm{N}), 132.1(\mathrm{Ph}), 130.1(\mathrm{Ph})$, $122.9(\mathrm{Ph}), 120.5(\mathrm{Ph}), 120.0(\mathrm{Py}), 105.5(\mathrm{Py}) \mathrm{ppm}$; MS (ESI) m/z: [(M + H $\left.{ }^{+}, 301.1\right]$. Anal. Calcd for $\mathrm{C}_{13} \mathrm{H}_{12} \mathrm{~N}_{6} \mathrm{O}_{3}$ : C, 52.00; H, 4.03; N, 27.99. Found: C, 52.35; H, 4.07; N, 27.72.

6-[2-(4-Methoxybenzylidene)hydrazinyl]nicotinohydrazide (4c). Yellow solid, yield 88\%, mp $244-246{ }^{\circ} \mathrm{C} ;{ }^{1} \mathrm{H}$ NMR (400.13 MHz, DMSO- $\left.d_{6}\right): \delta 10.92$ (s, 1H, NH), $9.50(\mathrm{~s}, 1 \mathrm{H}, \mathrm{NH}), 8.57$ (s, 1H, Py), 8.04 (s, 2H, Py + H), $7.61-7.63(\mathrm{~m}, 2 \mathrm{H}, \mathrm{Ph}), 7.19-7.22$ (m, 1H, Py), $6.96-6.98$ $(\mathrm{m}, 2 \mathrm{H}, \mathrm{Ph}), 4.37\left(\mathrm{~s}, 2 \mathrm{H}, \mathrm{NH}_{2}\right), 3.79\left(\mathrm{~s}, 3 \mathrm{H}, \mathrm{OCH}_{3}\right) \mathrm{ppm} ;{ }^{13} \mathrm{C} \mathrm{NMR}\left(100.61 \mathrm{MHz}, \mathrm{DMSO}-d_{6}\right): \delta$ $164.6(\mathrm{CO}), 159.8(\mathrm{Ph}), 158.2(\mathrm{Py}), 147.4(\mathrm{Py}), 140.5(\mathrm{Py}), 136.3(\mathrm{C}=\mathrm{N}), 127.5(\mathrm{Ph}), 127.4$ (Ph), $119.4(\mathrm{Py}), 114.0(\mathrm{Ph}), 104.0(\mathrm{Py}), 55.0\left(\mathrm{OCH}_{3}\right) \mathrm{ppm}$; MS (ESI) $m / z$ : [(M + H) $\left.{ }^{+}, 286.2\right]$. Anal. Calcd for $\mathrm{C}_{14} \mathrm{H}_{15} \mathrm{~N}_{5} \mathrm{O}_{2}$ : C, 58.94; H, 5.30; N, 24.55. Found: C, 58.88; H, 5.21; N, 24.43.

6-[2-(4-Methylbenzylidene)hydrazinyl]nicotinohydrazide (4d). White solid, yield 80\%, mp $274-276{ }^{\circ} \mathrm{C} ;{ }^{1} \mathrm{H}$ NMR (400.13 MHz, DMSO- $\left.d_{6}\right): \delta 11.14(\mathrm{~s}, 1 \mathrm{H}, \mathrm{NH}), 9.59(\mathrm{~s}, 1 \mathrm{H}, \mathrm{NH}), 8.57$ (s, 1H, Py), $8.02-8.04$ (m, 2H, Py + H), $7.57-7.59$ (m, 2H, Ph), $7.21-7.24$ (m, 3H, Ph + Py), 4.41 (s, 2H, NH ), 2.32 (s, 3H, $\mathrm{CH}_{3}$ ) ppm; ${ }^{13} \mathrm{C}$ NMR (100.61 MHz, DMSO- $\left.d_{6}\right): \delta 164.6(\mathrm{CO})$, $158.2(\mathrm{Py}), 147.4(\mathrm{Py}), 140.6(\mathrm{Py}), 138.3(\mathrm{C}=\mathrm{N}), 136.4(\mathrm{Ph}), 132.1(\mathrm{Ph}), 129.0(\mathrm{Ph}), 125.9(\mathrm{Ph})$, 
119.6 (Py), 105.0 (Py), $20.6\left(\mathrm{CH}_{3}\right)$ ppm; MS (ESI) $m / z$ : [(M + H) $\left.{ }^{+}, 270.1\right]$. Anal. Calcd for $\mathrm{C}_{14} \mathrm{H}_{15} \mathrm{~N}_{5} \mathrm{O}: \mathrm{C}, 62.44 ; \mathrm{H}, 5.61 ; \mathrm{N}, 26.01$. Found: C, 62.82; H, 5.53; N, 25.73.

6-[2-(4-Chlorobenzylidene)hydrazinyl]nicotinohydrazide (4e). White solid, yield 90\%, mp $280-282{ }^{\circ} \mathrm{C} ;{ }^{1} \mathrm{H}$ NMR (400.13 MHz, DMSO- $\left.d_{6}\right): \delta 11.30$ (s, $\left.1 \mathrm{H}, \mathrm{NH}\right), 9.62(\mathrm{~s}, 1 \mathrm{H}, \mathrm{NH}), 8.59$ (s, 1H, Py), $8.04-8.06(\mathrm{~s}, 2 \mathrm{H}, \mathrm{Py}+\mathrm{H}), 7.72(\mathrm{~d}, J=8,2 \mathrm{H}, \mathrm{Ph}), 7.47(\mathrm{~d}, J=8$, 2H, Ph), $7.26-$ 7.28 (m, 1H, Py), 4.37 (s, 2H, NH$)$ ppm; ${ }^{13} \mathrm{C}$ NMR (100.61 MHz, DMSO- $\left.d_{6}\right): \delta 164.6(\mathrm{CO})$, 158.1 (Py), 147.5 (Py), $139.2(\mathrm{Py}), 136.5(\mathrm{C}=\mathrm{N}), 133.7(\mathrm{Ph}), 133.0(\mathrm{Ph}), 128.5(\mathrm{Ph}), 127.5(\mathrm{Ph})$, 119.7 (Py), 105.2 (Py) ppm; MS (ESI) $m / z:\left[(\mathrm{M}+\mathrm{H})^{+}, 290.0\right]$. Anal. Calcd for $\mathrm{C}_{13} \mathrm{H}_{12} \mathrm{Cl} \mathrm{N}_{5} \mathrm{O}: \mathrm{C}$, 53.89; H, 4.17; N, 24.17. Found: C, 54.27; H, 4.15; N, 24.31.

6-[2-(Naphth-1-ylmethylidene)hydrazinyl]nicotinohydrazide (4f). Yellow solid, yield 85\%, mp $250-252{ }^{\circ} \mathrm{C} ;{ }^{1} \mathrm{H}$ NMR (400.13 MHz, DMSO-d $\left.d_{6}\right): \delta 11.36$ (s, 1H, NH), 9.68 (s, 1H, NH), 8.82 (s, 1H, Py), 8.65 (s, 1H, H), $8.14-8.15$ (m, 1H, Py), 7.95 - 8.09 (m, 4H, Naphthyl), 7.55 7.65 (m, 3H, Naphthyl), $7.30-7.34$ (m, 1H, Py), $4.48\left(\mathrm{~s}, 2 \mathrm{H}, \mathrm{NH}_{2}\right) \mathrm{ppm} ;{ }^{13} \mathrm{C}$ NMR (100.61 MHz, DMSO-d $\left.d_{6}\right): \delta 164.8(\mathrm{CO}), 158.4(\mathrm{Py}), 147.9(\mathrm{Py}), 140.3(\mathrm{Py}), 137.0(\mathrm{C}=\mathrm{N}), 133.6$ (Naphthyl), 130.1 (Naphthyl), 129.9 (Naphthyl), 129.4 (Naphthyl), 128.8 (Naphthyl), 127.1 (Naphthyl), 126.1 (Naphthyl), 125.9 (Naphthyl), 125.7 (Naphthyl), 123.6 (Naphthyl), 119.6 (Py), 105.4 (Py) ppm; MS (ESI) m/z: $\left[(\mathrm{M}+\mathrm{H})^{+}, 306.1\right]$. Anal. Calcd for $\mathrm{C}_{17} \mathrm{H}_{15} \mathrm{~N}_{5} \mathrm{O}: \mathrm{C}, 66.87 ; \mathrm{H}$, 4.95; N, 22.94. Found: C, 66.59; H, 4.93; N, 23.14.

6-[2-(Furan-2-ylmethylidene)hydrazinyl]nicotinohydrazide (4g). Brown solid, yield 64\%, mp 226 - $228{ }^{\circ} \mathrm{C} ;{ }^{1} \mathrm{H}$ NMR (400.13 MHz, DMSO-d $\left.)_{6}\right): \delta 11.20$ (s, 1H, NH), 9.57 (s, 1H, NH), 8.60 (s, 1H, Py), $8.01-8.07$ (m, 2H, Py + H), 7.74 (s, 1H, Furyl), $7.15-7.17$ (m, 1H, Furyl), $6.76-6.77$ (m, 1H, Py), $6.58-6.59$ (m, 1H, Furyl), 4.42 (s, 2H, $\left.\mathrm{NH}_{2}\right)$ ppm; ${ }^{13} \mathrm{C}$ NMR $(100.61 \mathrm{MHz}$, DMSO-d D) $\delta 164.6$ (CO), 158.0 (Py),149.9 (Furyl), 147.4 (Py), 143.7 (Py), 136.5 (C=N), 130.9 (Furyl),119.8 (Py), 111.7 (Furyl), 110.6 (Furyl), 105.0 (Py) ppm; MS (ESI) m/z: [(M + H) ${ }^{+}$, 246.0]. Anal. Calcd for $\mathrm{C}_{11} \mathrm{H}_{11} \mathrm{~N}_{5} \mathrm{O}_{2}$ : C, 53.87; H, 4.52; N, 28.56. Found: C, 54.22; H, 4.70; N, 28.84 .

General synthetic procedure for the synthesis of 5-[(3-alkyl(aryl/heteroaryl)-5trifluoromethyl-5-hydroxy-4,5-dihydro-1H-pyrazol-1-yl)carbonylpyridin-2-yl]aryl/-

heteroarylaldehyde hydrazones (6aa - 6ac, 6ba - 6bc, 6ca - 6cc). A mixture of 1,1,1trifluoro-4-alkyl(aryl/heteroaryl)-4-methoxy-3-alken-2-one $\quad(\mathbf{5 a - c}) \quad(1 \mathrm{mmol})$ and 6-[2aryl/heteroarylmethylidenehydrazino]nicotinic hydrazide (4a-c) $(1 \mathrm{mmol})$ in $15 \mathrm{~cm}^{3}$ of ethanol was refluxed under stirring for $4 \mathrm{~h}$. Products 6aa-6cc were obtained as solids, filtered under reduced pressure, and dried in a desiccator containing $\mathrm{P}_{2} \mathrm{O}_{5}$ under vacuum. All compounds presented a high degree of purity and did not undergo recrystallization.

5-[(5-Trifluoromethyl-5-hydroxy-3-methyl-4,5-dihydro-1 $H$-pyrazol-1-yl)- carbonylpyridin2-yl]benzaldehyde hydrazine (6aa). White solid, yield 79\%, mp $102-104{ }^{\circ} \mathrm{C}$; ${ }^{1} \mathrm{H}$ NMR (400.13 MHz, DMSO-d $d_{6}$ ): $\delta 11.34$ (s, 1H, NH), 8.57 (s, 1H, Py), 8.11 (s, 1H, H), 8.01 - 8.06 (m, 1H, Py), $7.68-7.79$ (m, 3H, Ph), $7.39-7.43$ (m, 2H, Ph), $7.25-7.30$ (m, 1H, Py), 3.49 (d, 1H, $\left.J_{\mathrm{H} 4-\mathrm{H} 4}, 19 \mathrm{~Hz}, 1 \mathrm{H}, \mathrm{H} 4-\mathrm{Pz}\right), 3.09$ (d, 1H, $\left.J_{\mathrm{H} 4-\mathrm{H} 4}, 19 \mathrm{~Hz}, 1 \mathrm{H}, \mathrm{H} 4{ }^{\prime}-\mathrm{Pz}\right), 2.02\left(\mathrm{~s}, 3 \mathrm{H}, \mathrm{CH}_{3}\right) \mathrm{ppm} ;{ }^{13} \mathrm{C}$ 
NMR (100.61 MHz, DMSO-d $\left.d_{6}\right): \delta 165.1$ (CO), 157.9 (Py), 154.2 (C3-Pz), 150.2 (Py), 149.9 $(\mathrm{C}=\mathrm{N}), 143.9(\mathrm{Py}), 139.9(\mathrm{Ph}), 131.5(\mathrm{Ph}), 123.2$ (q, $\left.{ }^{1} J 286 \mathrm{~Hz}, \mathrm{CF}_{3}\right), 120.9(\mathrm{Py}), 111.8(\mathrm{Ph})$, $110.9(\mathrm{Ph}), 104.5(\mathrm{Py}), 91.5$ (q, $\left.{ }^{1} J 33 \mathrm{~Hz}, \mathrm{C} 5-\mathrm{Pz}\right), 47.2\left(\mathrm{CH}_{2}-\mathrm{Pz}\right), 15.0\left(\mathrm{CH}_{3}\right) \mathrm{ppm}$; MS (ESI) $m / z:\left[(\mathrm{M}+\mathrm{H})^{+}\right.$, 392.1]. Anal. Calcd for $\mathrm{C}_{18} \mathrm{H}_{16} \mathrm{~F}_{3} \mathrm{~N}_{5} \mathrm{O}_{2}$ : C, 55,24; H, 4.12; N, 17.90. Found: $\mathrm{C}$, $55.46 ; \mathrm{H}, 4.43 ; \mathrm{N}, 17.58$.

5-[(5-Trifluoromethyl-5-hydroxy-3-phenyl-4,5-dihydro-1 $H$-pyrazol-1-yl)- carbonylpyridin2-yl]benzaldehyde hydrazone (6ab). White solid, yield $58 \%$, mp $219-221{ }^{\circ} \mathrm{C}$; ${ }^{1} \mathrm{H}$ NMR $\left(400.13 \mathrm{MHz}, \mathrm{DMSO}-d_{6}\right): \delta 11.38(\mathrm{~s}, 1 \mathrm{H}, \mathrm{NH}), 8.69$ (s, 1H, Py), $8.09-8.21(\mathrm{~m}, 2 \mathrm{H}, \mathrm{Py}+\mathrm{H})$, $7.72-7.79(\mathrm{~m}, 5 \mathrm{H}, \mathrm{Ph}), 7.33-7.51(\mathrm{~m}, 6 \mathrm{H}, \mathrm{Ph}+\mathrm{Py}), 3.96\left(\mathrm{~d}, 1 \mathrm{H}, J_{\mathrm{H} 4-\mathrm{H} 4}=19 \mathrm{~Hz}, 1 \mathrm{H}, \mathrm{H} 4-\mathrm{Pz}\right)$, $3.62\left(\mathrm{~d}, 1 \mathrm{H}, J_{\mathrm{H} 4-\mathrm{H} 4}=19 \mathrm{~Hz}, 1 \mathrm{H}, \mathrm{H} 4{ }^{\prime}-\mathrm{Pz}\right) \mathrm{ppm} ;{ }^{13} \mathrm{C} \mathrm{NMR}\left(100.61 \mathrm{MHz}, \mathrm{DMSO}-d_{6}\right): \delta 164.9$ (CO), 158.1 (Py), 151.7 (C3-Pz), $150.4(\mathrm{Py}), 141.2(\mathrm{C}=\mathrm{N}), 139.3(\mathrm{Py}), 134.7(\mathrm{Ph}), 130.3(\mathrm{Ph})$, $129.9(\mathrm{Ph}), 128.7(\mathrm{Ph}), 128.5(\mathrm{Ph}), 128.4(\mathrm{Ph}), 126.3(\mathrm{Ph}), 126.0(\mathrm{Ph}), 123.1\left(\mathrm{q},{ }^{1} J 286 \mathrm{~Hz}, \mathrm{CF}_{3}\right)$, 120.7 (Py), 104.6 (Py), 92.1 (q, $\left.{ }^{1} J 33 \mathrm{~Hz}, \mathrm{C} 5-\mathrm{Pz}\right), 43.6\left(\mathrm{CH}_{2}-\mathrm{Pz}\right) \mathrm{ppm}$; MS (ESI) m/z: [(M + $\mathrm{H})^{+}$, 454.2]. Anal. Calcd for $\mathrm{C}_{23} \mathrm{H}_{18} \mathrm{~F}_{3} \mathrm{~N}_{5} \mathrm{O}_{2}$ : C, 60.93; H, 4.00; N, 15.45. Found: C, 61.31; H, $4.21 ; \mathrm{N}, 15.71$.

5-[(5-Trifluoromethyl-5-hydroxy-3-(2-furyl)-4,5-dihydro-1H-pyrazol-1-yl)carbonylpyridin-2-yl]benzaldehyde hydrazone (6ac). Yellow solid, yield 45\%; mp $212-214$ ${ }^{\circ} \mathrm{C} ;{ }^{1} \mathrm{H}$ NMR (400.13 MHz, DMSO-d $)$ ) $\delta 11.18$ (s, 1H, NH), 8.66 (s, 1H, Py), $8.10-8.14$ (m, $2 \mathrm{H}, \mathrm{Py}+\mathrm{H}), 7.86(\mathrm{~s}, 1 \mathrm{H}$, Furyl), $7.70-7.71(\mathrm{~m}, 2 \mathrm{H}, \mathrm{Ph}), 7.33-7.44(\mathrm{~m}, 3 \mathrm{H}, \mathrm{Ph}), 7.29-7.31$ (m, 1H, Furyl), 7.06 (s, 1H, Furyl), $6.65-6.67$ (m, 1H, Py), 3.82 (d, 1H, $J_{\mathrm{H} 4-\mathrm{H} 4}, 18 \mathrm{~Hz}, \mathrm{H} 4-\mathrm{Pz}$ ), 3.49 (d, 1H, $\left.J_{\mathrm{H} 4-\mathrm{H} 4}, 18 \mathrm{~Hz}, 1 \mathrm{H}, \mathrm{H} 4{ }^{\prime}-\mathrm{Pz}\right) \mathrm{ppm} .{ }^{13} \mathrm{C}$ NMR (100.61 MHz, DMSO- $\left.d_{6}\right): \delta 164.8(\mathrm{CO})$, 158.2 (Py), 150.5 (C3-Pz), 145.7 (Py), $145.1(\mathrm{C}=\mathrm{N}), 143.1$ (Py), 141.2 (Furyl), 139.5 (Furyl), $134.8(\mathrm{Ph}), 128.9(\mathrm{Ph}), 128.6(\mathrm{Ph}), 126.2(\mathrm{Ph}), 125.9$ (q, $\left.{ }^{1} J 286 \mathrm{~Hz}, \mathrm{CF}_{3}\right), 120.7(\mathrm{Py}), 114.6$ (Furyl), 112.1 (Furyl), 104.7 (Py), 91.7 (q, $\left.{ }^{1} J 33 \mathrm{~Hz}, \mathrm{C} 5-\mathrm{Pz}\right), 43.5\left(\mathrm{CH}_{2}-\mathrm{Pz}\right) \mathrm{ppm}$. MS (ESI) m/z: $\left[(\mathrm{M}+\mathrm{H})^{+}\right.$, 444.1]. Anal. Calcd for $\mathrm{C}_{21} \mathrm{H}_{16} \mathrm{~F}_{3} \mathrm{~N}_{5} \mathrm{O}_{3}: \mathrm{C}, 56.89 ; \mathrm{H}, 3.64 ; \mathrm{N}, 15.80$. Found: C, 57.07; $\mathrm{H}, 3.88 ; \mathrm{N}, 16.03$.

5-[(5-Trifluoromethyl-5-hydroxy-3-methyl-4,5-dihydro-1H-pyrazol-1-yl)- carbonylpyridin2-yl]3-nitrobenzaldehyde hydrazone (6ba). Yellow solid, yield 73\%, mp $246-248{ }^{\circ} \mathrm{C} ;{ }^{1} \mathrm{H}$ NMR (400.13 MHz, DMSO-d $): \delta 11.59$ (s, 1H, NH), $8.59-8.60$ (m, 1H, Py), 8.49 (s, 1H, H), 8.07 - 8.22 (m, 2H, Ph), 8.07 (s, 1H, Py), 7.80 (s, 1H, Ph), $7.69-7.73$ (m, 1H, Ph), 7.33 - 7.35 (m, 1H, Py), 3.49 (d, 1H, J J4-H4 19 Hz, H4-Pz), 3.11 (d, 1H, J J4-H4 19 Hz, 1H, H4'-Pz), 2.03 (s, $\left.3 \mathrm{H}, \mathrm{CH}_{3}\right) \mathrm{ppm} ;{ }^{13} \mathrm{C}$ NMR (100.61 MHz, DMSO- $\left.d_{6}\right): \delta 164.9(\mathrm{CO}), 157.7(\mathrm{Py}), 154.1(\mathrm{C} 3-\mathrm{Pz})$, $150.1(\mathrm{Py}), 148.1(\mathrm{C}=\mathrm{N}), 139.3(\mathrm{Py}), 138.4(\mathrm{Ph}), 136.7(\mathrm{Ph}), 132.0(\mathrm{Ph}), 129.9(\mathrm{Ph}), 123.2\left(\mathrm{q},{ }^{1} J\right.$ $286 \mathrm{~Hz}, \mathrm{CF}_{3}$ ), $122.7(\mathrm{Ph}), 121.5(\mathrm{Ph}), 119.9(\mathrm{Py}), 104.9$ (Py), 91.4 (q, $\left.{ }^{1} J 33 \mathrm{~Hz}, \mathrm{C} 5-\mathrm{Pz}\right), 47.1$ $\left(\mathrm{CH}_{2}-\mathrm{Pz}\right), 15.0\left(\mathrm{CH}_{3}\right)$ ppm; MS (ESI) $\mathrm{m} / z:\left[(\mathrm{M}+\mathrm{H})^{+}\right.$, 437.0]. Anal. Calcd for $\mathrm{C}_{18} \mathrm{H}_{15} \mathrm{~F}_{3} \mathrm{~N}_{6} \mathrm{O}_{4}$ : C, 49.55; H, 3.46; N, 19.26. Found: C, 49.90; H, 3.21; N, 19.42.

5-[(5-Trifluoromethyl-5-hydroxy-3-phenyl-4,5-dihydro-1H-pyrazol-1-yl)-carbonylpyridin-

2-yl]3-nitrobenzaldehyde hydrazone (6bb). Yellow solid, yield $76 \%$, mp $263-265{ }^{\circ} \mathrm{C} ;{ }^{1} \mathrm{H}$ NMR (400.13 MHz, DMSO-d $): \delta 11.67$ (s, 1H, NH), $8.69-8.70$ (m, 1H, Py), 8.51 (s, 1H, H), $8.15-8.24(\mathrm{~m}, 5 \mathrm{H}, \mathrm{Ph}), 7.76-7.80(\mathrm{~m}, 2 \mathrm{H}, \mathrm{Ph}), 7.76-7.80(\mathrm{~m}, 2 \mathrm{H}, \mathrm{Ph}), 7.68-7.72(\mathrm{~m}, 1 \mathrm{H}$, 
Py), 7.49 - 7.52 (m, 2H, Ph),7.39 - 7.43 (m, 1H, Py), 3.97 (d, 1H, J J4-H4, 19 Hz, 1H, H4-Pz), $3.63\left(\mathrm{~d}, 1 \mathrm{H}, J_{\mathrm{H} 4-\mathrm{H} 4}, 19 \mathrm{~Hz}, \mathrm{H} 4{ }^{\prime}-\mathrm{Pz}\right) \mathrm{ppm} ;{ }^{13} \mathrm{C}$ NMR (100.61 MHz, DMSO-d $\left.d_{6}\right): \delta 164.7$ (CO), 157.7 (Py), 151.7 (C3-Pz), 150.2 (Py), $148.1(\mathrm{C}=\mathrm{N}), 139.4(\mathrm{Py}), 138.5(\mathrm{Ph}), 136.6(\mathrm{Ph}), 132.0$ $(\mathrm{Ph}), 130.3(\mathrm{Ph}), 129.9(\mathrm{Ph}), 128.5(\mathrm{Ph}), 126.3(\mathrm{Ph}), 123.6(\mathrm{Ph}), 123.0\left(\mathrm{q},{ }^{1} J 285 \mathrm{~Hz}, \mathrm{CF}_{3}\right), 122.7$ (Ph), $121.4(\mathrm{Ph}), 119.9$ (Py), 104.9 (Py), 92.1 (q, $\left.{ }^{1} J 33 \mathrm{~Hz}, \mathrm{C} 5-\mathrm{Pz}\right), 43.6\left(\mathrm{CH}_{2}-\mathrm{Pz}\right) \mathrm{ppm}$; MS (ESI) $m / z:\left[(\mathrm{M}+\mathrm{H})^{+}, 499.0\right]$. Anal. Calcd for $\mathrm{C}_{23} \mathrm{H}_{17} \mathrm{~F}_{3} \mathrm{~N}_{6} \mathrm{O}_{4}$ : C, 52.68; H, 3.45; N, 17.98 . Found: C, 52.34; H, 3.44; N, 17.65.

\section{5-[(5-Trifluoromethyl-5-hydroxy-3-(2-furyl)-4,5-dihydro-1H-pyrazol-1-yl)-}

carbonylpyridin-2-yl]3-nitrobenzaldehyde hydrazone (6bc). Yellow solid, yield 83\%, mp 252 $-254{ }^{\circ} \mathrm{C} ;{ }^{1} \mathrm{H}$ NMR $\left(400.13 \mathrm{MHz}, \mathrm{DMSO}-d_{6}\right): \delta 11.48(\mathrm{~s}, 1 \mathrm{H}, \mathrm{NH}), 8.67-8.68(\mathrm{~m}, 1 \mathrm{H}, \mathrm{Py}), 8.48$ (s, 1H, H), 8.24 (s, 1H, Py), $8.13-8.16$ (m, 3H, Ph), 7.87 (s, 1H, Furyl), $7.68-7.72$ (m, 1H, Ph), $7.35-7.37$ (m, 1H, Furyl), $7.07-7.08$ (m, 1H, Py), $6.66-6.67$ (m, 1H, Furyl), 3.83 (d, 1H, $J_{\mathrm{H} 4-}$ $\mathrm{H} 4=18 \mathrm{~Hz}, \mathrm{H} 4-\mathrm{Pz}), 3.50$ (d, 1H, $\left.J_{\mathrm{H} 4-\mathrm{H} 4}=18 \mathrm{~Hz}, \mathrm{H} 4{ }^{\prime}-\mathrm{Pz}\right) \mathrm{ppm} ;{ }^{13} \mathrm{C}$ NMR $(100.61 \mathrm{MHz}, \mathrm{DMSO}-$ $\left.d_{6}\right): \delta 164.6(\mathrm{CO}), 157.8(\mathrm{Py}), 150.2(\mathrm{C} 3-\mathrm{Pz}), 148.1(\mathrm{Py}), 145.5(\mathrm{C}=\mathrm{N}), 145.0(\mathrm{Py}), 143.1$ (Furyl), 139.4 (Furyl), $138.6(\mathrm{Ph}), 136.6(\mathrm{Ph}), 132.0(\mathrm{Ph}), 129.9(\mathrm{Ph}), 122.7(\mathrm{Ph}), 122.9\left(\mathrm{q},{ }^{1} J\right.$ $286 \mathrm{~Hz}, \mathrm{CF}_{3}$ ), 121.6 (Ph), 119.9 (Py), 114.2 (Furyl), 111.9 (Furyl) 104.9 (Py), 91.6 (q, ${ }^{1} J 33 \mathrm{~Hz}$, C5-Pz), $43.4\left(\mathrm{CH}_{2}-\mathrm{Pz}\right)$ ppm; MS (ESI) $m / z$ : $\left[(\mathrm{M}+\mathrm{H})^{+}\right.$, 489.1]. Anal. Calcd for $\mathrm{C}_{21} \mathrm{H}_{15} \mathrm{~F}_{3} \mathrm{~N}_{6} \mathrm{O}_{5}$ : C, 52.33; H, 3.33; N, 17.72. Found: C, 52.37; H, 3.16; N, 17.63.

\section{5-[(5-Trifluoromethyl-5-hydroxy-3-methyl-4,5-dihydro-1 $H$-pyrazol-1-yl)-carbonylpyridin-}

2-yl]4-methoxybenzaldehyde hydrazone (6ca). White solid, yield $77 \%$, mp $194-196{ }^{\circ} \mathrm{C} ;{ }^{1} \mathrm{H}$ NMR (400.13 MHz, DMSO- $\left.d_{6}\right): \delta 11.10(\mathrm{~s}, 1 \mathrm{H}, \mathrm{NH}), 8.56(\mathrm{~s}, 1 \mathrm{H}, \mathrm{Py}), 7.99-8.06$ (m, 2H, Py + $\mathrm{H}), 7.63(\mathrm{~d}, J$ 8, 2H, Ph), $7.19-7.24$ (m, 1H, Py), 6.98 (d, J 8, 2H, Ph), 3.8 (s, 3H, OCH $), 3.47$ (d, 1H, $\left.J_{\mathrm{H} 4-\mathrm{H} 4}, 19 \mathrm{~Hz}, \mathrm{H} 4-\mathrm{Pz}\right), 3.10$ (d, 1H, $J_{\mathrm{H} 4-\mathrm{H} 4}, 19 \mathrm{~Hz}, 1 \mathrm{H}, \mathrm{H} 4$ '-Pz), 2.02 (s, 3H, CH ${ }_{3}$ ppm; ${ }^{13} \mathrm{C}$ NMR (100.61 MHz, DMSO- $\left.d_{6}\right)$ : $\delta 165.0(\mathrm{CO}), 160.0(\mathrm{Py}), 158.2(\mathrm{C} 3-\mathrm{Pz}), 154.2(\mathrm{Ph}), 150.4$ (Py), $141.1(\mathrm{C}=\mathrm{N}), 139.3(\mathrm{Py}), 127.7(\mathrm{Ph}), 123.2$ (q, $\left.{ }^{1} J=286 \mathrm{~Hz}, \mathrm{CF}_{3}\right), 120.4(\mathrm{Py}), 114.1(\mathrm{Ph})$, 104.4 (Py), 91.4 (q, $\left.{ }^{1} J=33 \mathrm{~Hz}, \mathrm{C} 5-\mathrm{Pz}\right), 55.1\left(\mathrm{OCH}_{3}\right), 47.2\left(\mathrm{CH}_{2}-\mathrm{Pz}\right), 15.1\left(\mathrm{CH}_{3}\right) \mathrm{ppm}$; MS (ESI) $m / z:\left[(\mathrm{M}+\mathrm{H})^{+}, 422.1\right]$. Anal. Calcd for $\mathrm{C}_{18} \mathrm{H}_{18} \mathrm{~F}_{3} \mathrm{~N}_{5} \mathrm{O}_{3}: \mathrm{C}, 54.16 ; \mathrm{H}, 4.31 ; \mathrm{N}, 16.62$. Found: $\mathrm{C}$, 54.35; H, 4.29; N, 16.58 .

\section{5-[(5-Trifluoromethyl-5-hydroxy-3-phenyl-4,5-dihydro-1H-pyrazol-1-yl)-carbonylpyridin-}

2-yl]4-methoxybenzaldehyde hydrazone (6cb). White solid, yield 79\%, mp $217-219{ }^{\circ} \mathrm{C} ;{ }^{1} \mathrm{H}$ NMR (400.13 MHz, DMSO- $d_{6}$ ): $\delta 11.09$ (s, 1H, NH), 8.68 (s, 1H, Py), $8.14-8.17$ (m, 1H, Py), 8.10 (s, 1H, H), $7.75-7.77$ (m, 2H, Ph), 7.66 (d, J 8, 2H, Ph), $7.48-7.49$ (m, 3H, Ph), 7.28 - 7.30 (m, 1H, Py), 6.99 (d, J 8, 2H, Ph), 3.92 (d, 1H, JH4-H4 19 Hz, H4-Pz), 3.80 (s, 3H, OCH $)_{3}$, $3.61\left(\mathrm{~d}, 1 \mathrm{H}, J_{\mathrm{H} 4-\mathrm{H} 4}, 19 \mathrm{~Hz}, \mathrm{H} 4{ }^{\prime}-\mathrm{Pz}\right) \mathrm{ppm} ;{ }^{13} \mathrm{C} \mathrm{NMR}\left(100.61 \mathrm{MHz}, \mathrm{DMSO}-d_{6}\right): \delta 165.0(\mathrm{CO})$, 160.0 (Py), 158.2 (C3-Pz), $151.7(\mathrm{Ph}), 150.6(\mathrm{Py}), 141.3(\mathrm{C}=\mathrm{N}), 139.4(\mathrm{Py}), 130.4(\mathrm{Ph}), 130.0$ $(\mathrm{Ph}), 128.6(\mathrm{Ph}), 127.7(\mathrm{Ph}), 127.4(\mathrm{Ph}), 126.4(\mathrm{Ph}), 123.2\left(\mathrm{q},{ }^{1} J=286 \mathrm{~Hz}, \mathrm{CF}_{3}\right), 120.3(\mathrm{Py})$, $114.1(\mathrm{Ph}), 104.5(\mathrm{Py}), 92.2$ (q, $\left.{ }^{1} \mathrm{~J}=33 \mathrm{~Hz}, \mathrm{C} 5-\mathrm{Pz}\right), 55.0\left(\mathrm{OCH}_{3}\right), 43.7\left(\mathrm{CH}_{2}-\mathrm{Pz}\right) \mathrm{ppm}$; MS (ESI) $m / z:\left[(\mathrm{M}+\mathrm{H})^{+}\right.$, 484.1]. Anal. Calcd for $\mathrm{C}_{24} \mathrm{H}_{20} \mathrm{~F}_{3} \mathrm{~N}_{5} \mathrm{O}_{3}: \mathrm{C}, 59.63 ; \mathrm{H}, 4.17 ; \mathrm{N}, 14.49$. Found: $\mathrm{C}$, 59.94; H, 4.21; N, 14.64. 


\section{5-[(5-Trifluoromethyl-5-hydroxy-3-(2-furyl)-4,5-dihydro-1H-pyrazol-1-yl)-}

carbonylpyridin-2-yl]4-methoxybenzaldehyde hydrazone (6cc). White solid, yield 76\%, mp $226-228{ }^{\circ} \mathrm{C}$; ${ }^{1} \mathrm{H}$ NMR (400.13 MHz, DMSO- $d_{6}$ ): $\delta 11.10$ (s, 1H, NH), 8.64 (s, 1H, Py), $8.07-$ $8.10(\mathrm{~m}, 2 \mathrm{H}, \mathrm{Py}+\mathrm{H}), 7.72-7.73(\mathrm{~m}, 1 \mathrm{H}$, Furyl), $7.65(\mathrm{~d}, J$ 8, 2H, Ph), $7.55-7.56(\mathrm{~m}, 1 \mathrm{H}$, Furyl), $7.24-7.28$ (m, 1H, Furyl), $7.16-7.18$ (m, 1H, Py), 6.99 (d, J 8, 2H, Ph), 3.93 (d, 1H, $\left.J_{\mathrm{H} 4-\mathrm{H} 4}=18 \mathrm{~Hz}, \mathrm{H} 4-\mathrm{Pz}\right), 3.80\left(\mathrm{~s}, 3 \mathrm{H}, \mathrm{OCH}_{3}\right), 3.59$ (d, 1H, $\left.J_{\mathrm{H} 4-\mathrm{H} 4}, 19 \mathrm{~Hz}, \mathrm{H} 4{ }^{\prime}-\mathrm{Pz}\right) \mathrm{ppm} ;{ }^{13} \mathrm{C}$ NMR (100.61 MHz, DMSO-d $)_{6}$ : $\delta 164.4$ (CO), 159.9 (Py), 158.1 (C3-Pz), 150.5 (Ph), 147.5 (Py), $141.2(\mathrm{C}=\mathrm{N}), 139.3$ (Py), 133.0 (Furyl), 130.6 (Furyl), 129.7 (Furyl), 128.3 (Furyl), 127.9 (Ph), 127.6 (Ph), 123.0 (q, ${ }^{1} J 286$ Hz, CF 3 ), 120.1 (Py), 114.0 (Ph), 104.3 (Py), 92.1 (q, ${ }^{1} J 33$ Hz, C5$\mathrm{Pz}), 55.4\left(\mathrm{OCH}_{3}\right), 44.2\left(\mathrm{CH}_{2}-\mathrm{Pz}\right) \mathrm{ppm}$; MS (ESI) $\mathrm{m} / \mathrm{z}:\left[(\mathrm{M}+\mathrm{H})^{+}, 474.2\right]$. Anal. Calcd for $\mathrm{C}_{22} \mathrm{H}_{18} \mathrm{~F}_{3} \mathrm{~N}_{5} \mathrm{O}_{4}$ : C, 55.82; H, 3.83; N, 14.79. Found: C, 56.17; H, 4.21; N, 14.46.

General synthetic procedure for the synthesis of 2-(5-(5-methyl-1,3,4-oxadiazol-2-yl)pyridin-2-yl)-4-chlorobenzaldehyde hydrazone (7e). A mixture of 6-[2aryl/heteroarylmethylidenehydrazino]nicotinic hydrazide (4e) $(1 \mathrm{mmol})$ and triethylortoacetate $\left(1.1 \mathrm{~cm}^{3}, 6 \mathrm{mmol}\right)$ was stirred for $16 \mathrm{~h}$ at reflux. The excess of orthoacetate was removed by evaporation under reduced pressure. The product 7e was obtained as solid and dried under vacuum in a desiccator containing $\mathrm{P}_{2} \mathrm{O}_{5}$.

2-(5-(5-Methyl-1,3,4-oxadiazol-2-yl)-pyridin-2-yl)-4-chlorobenzaldehyde hydrazone (7e) Brown solid, yield 64\%, mp $161-163{ }^{\circ} \mathrm{C} ;{ }^{1} \mathrm{H}$ NMR (400.13 MHz, DMSO- $\left.d_{6}\right): \delta 11.35(\mathrm{~s}, 1 \mathrm{H}$, $\mathrm{NH}), 8.68$ (s, 1H, Py), $8.12-8.14$ (m, 2H Py + H), $7.70-7.73(\mathrm{~m}, 2 \mathrm{H}, \mathrm{Ph}), 7.44-7.47$ (m, 2H, $\mathrm{Ph}), 7.37-7.40(\mathrm{~m}, 1 \mathrm{H}, \mathrm{Py}), 2.55\left(\mathrm{~s}, 3 \mathrm{H}, \mathrm{CH}_{3}\right) \mathrm{ppm} ;{ }^{13} \mathrm{C}$ NMR (100.61 MHz, DMSO- $\left.d_{6}\right): \delta$ $162.8\left(\mathrm{C}_{5}-\mathrm{Oxz}\right), 162.5\left(\mathrm{C}_{2}-\mathrm{Oxz}\right), 158.2(\mathrm{Py}) ; 146.4(\mathrm{Py}), 139.9(\mathrm{Py}), 135.5(\mathrm{C}=\mathrm{N}), 128.6(\mathrm{Ph})$, $128.5(\mathrm{Ph}), 127.7(\mathrm{Ph}), 127.5(\mathrm{Ph}), 119.8(\mathrm{Py}), 106.3(\mathrm{Py}), 10.3\left(\mathrm{CH}_{3}-\mathrm{Oxz}\right) \mathrm{ppm}$; MS (ESI) m/z: $\left[(\mathrm{M}+\mathrm{H})^{+}, 314.1\right]$. Anal. Calcd for $\mathrm{C}_{15} \mathrm{H}_{12} \mathrm{Cl} \mathrm{N}{ }_{5} \mathrm{O}: \mathrm{C}, 57.42 ; \mathrm{H}, 3.86 ; \mathrm{N}, 22.32$. Found: C, 57.39; $\mathrm{H}, 4.01 ; \mathrm{N}, 22.65$.

\section{Acknowledgements}

The authors thank the financial support from Conselho Nacional de Desenvolvimento Científico e Tecnológico - CNPq (Proc. 470788/2010-0 Universal). Fellowships from CAPES and CNPq are also acknowledged.

\section{References}

1. Angelusiu, M. V.; Barbuceanu, S. F., Draghici, C.; Almajan, G. L. Eur. J. Med. Chem. 2010, 45, 2055.

2. Belskaya, N. P.; Dehaen, W.; Bakulev, V. A. Arkivoc 2010, (i), 275.

3. Rollas, S. Kucukguzel, S. G. Molecules 2007, 12, 1910. 
4. Ozkay, Y.; Tunah, Y.; Karaca, H.; Isikdag, I. Eur. J. Med. Chem. 2010, 45, 3293.

5. Candéa, A. L. P.; Ferreira, M. L.; Pais, K. C.; Cardoso, L. N. F.; Kaiser, C. R.; Henriques, M. G. M. O.; Lourenço, M. C. S.; Bezerra, F. A. F. M.; Souza, M. V. N. Bioorg. Med. Chem. Lett. 2009, 19, 6272.

6. Joshi, S. D.; Vagdevi, H. M.; Vaidya, V. P.; Gadaginamath, G. S. Eur. J. Med. Chem. 2008, $43,1989$.

7. Sreeja, P. B.; Sreekanth, A.; Nayar, C. R.; Kurup, M. R. P.; Usman, A.; Rasaz, I. A.; Chantrapromma, S.; Fun, H. K. J. Mol. Struct. 2003, 645, 221.

8. Kuriakose, M.; Kurup, M. R. P.; Suresh, E. Spectrochim. Acta Part A 2007, 66, 353.

9. Sauzem, P. D.; Machado, P.; Rubin, M. A.; Sant'Anna, G. S.; Faber, H. B.; Souza, A. H.; Mello, C. F.; Beck, P.; Burrow, R. A.; Bonacorso, H. G.; Zanatta, N.; Martins, M. A. P. Eur. J. Med. Chem. 2008, 43, 1237.

10. Bonacorso, H. G.; Cunico, W.; Cechinel, C. A.; Martins, M. A. P.; Zanatta. N.; Souza, M.; Freitas, I.; Soares, R.; Kretlli, A. Bioorg. Med. Chem. Lett. 2006, 16, 649.

11. Bekhit, A. A.; Abdel-Aziem, T. Bioorg. Med. Chem. 2004, 12, 1936.

12. Zora, M.; Gormen, M. J. Organomet. Chem. 2007, 692, 5026.

13. Xia, Y.; Dong, Z. W.; Zhao, B. X.;Ge, X.; Meng, N.; Shin, D. S.; Miao, J. Y. Bioorg. Med. Chem. 2007, 15, 6893.

14. Park, D.Y.; Lee, M. J.; Kim, T.H. Tetrahedron Lett. 2005, 46, 8799.

15. Bonacorso, H. G.; Paim, G. R.; Guerra, C. Z.; Sehnem, R. C.; Cechinel, C. A.; Porte, L. M. F.; Martins, M. A. P.; Zanatta, N. J. Braz. Chem. Soc. 2009, 20, 509.

16. Ainsworth, C. J. Am. Chem. Soc. 1955, 77, 1148.

17. Xia, Y.; Fan, C. D.; Zhao, B. X.; Zhao, J.; Shin, D. S.; Miao, J. Y. Eur. J. Med. Chem. 2008, $43,2347$.

18. Du, X.; Guo, C.; Hansell, E.; Doyle, P. S.; Caffrey, C. R.; Holler, T. P.; McKerrow, J. H.; Cohen, F. E. J. Med. Chem. 2002, 45, 2695.

19. Kumar, R.; Nair, R. R.; Dhiman, S. S.; Prakash, O. Eur. J. Med. Chem. 2009, 44, 2260.

20. Effenberger, F.; Maier, R.; Schonwalder, K. H.; Ziegler, T. Chem. Ber. 1982, 115, 2766.

21. Siqueira, G. M.; Flores, A. F. C.; Clar, G.; Zanatta, N. Martins, M. A. P. Quim. Nova 1994, $17,24$.

22. Druzhinin, S. V.; Balenkova, E. S.; Nenajdenko, V. G. Tetrahedron 2007, 63, 7753.

23. Martins, M. A. P.; Guarda, E.; Frizzo, C. P.; Scapin, E.; Beck, P.; Costa, A. C.; Zanatta, N.; Bonacorso, H. G. J. Mol. Catal. A 2007, 266, 100. 